\title{
Utilidade do pente metálico com dentes microcanaliculados no diagnóstico da pediculose
}

\author{
Metal microchanelled fine-toothed comb use in the diagnosis of pediculosis \\ Patricia Elena Neira ${ }^{1}$ \\ Alejandra Ximena Correa ${ }^{3}$ \\ Luis Rodrigo Molina ${ }^{2}$ \\ Nelson Ramón Américo Muñoz ${ }^{4}$
}

David Eduardo Oschilewski ${ }^{5}$

\begin{abstract}
Resumo: FundAMENTOS: Os métodos para diagnosticar Pediculus humanus var. capitis são controvertidos e a maioria dos estudos tem usado o exame visual direto.

OBjetrvos: O objetivo deste estudo foi comparar a eficácia no diagnóstico do exame visual direto em contraposição ao uso do pente metálico de aço com dentes microcanaliculados.

Métodos: Investigaram-se 946 crianças e adolescentes de quatro a 19 anos. O cabelo de cada criança foi examinado duas vezes, para detectar infestação pelos diferentes estádios, por meio da observação visual direta e com o pente metálico.

RESUltADOS: O diagnóstico visual apontou infestação em 30,7\% dos casos, enquanto que o pente metálico indicou um resultado de $51,5 \%$. O sexo feminino foi o mais acometido, de acordo com ambos os métodos. As formas parasitárias detectadas com o exame visual direto foram: somente piolhos (adultos e/ou ninfas); $1,4 \%$; somente lêndeas vivas, $64,8 \%$; e lêndeas vivas mais piolhos, $33,8 \%$; com o pente metálico, foram $6,4 \%$, $23,6 \%$ e $70 \%$, respectivamente. A média de tempo para descobrir um piolho foi de 57 segundos com o pente metálico e de 116,4 segundos com a observação visual direta.

CONCLUSÕEs: O diagnóstico é duas vezes mais rápido e 3,6 vezes mais eficiente com o pente metálico microcanaliculado. O método visual leva a uma subestimação das infestações ativas e revela as passadas não ativas. Palavras-chave: Chile; dermatoses do couro cabeludo; infestações por piolhos; Pediculus capitis
\end{abstract}

\begin{abstract}
BACKGROUnD: Methods for the diagnosis of Pediculus humanus var. capitis are controversial and most studies are based on direct visual exam.

ОвјестіvE: The objective of this study was to compare the diagnosis efficacy of both direct visual exam and the use of a metal microchanelled fine-toothed comb.

METHODS: 946 children and teenagers 4 to 19 years of age were examined. Each individual's hair was examined twice to determine whether there was infestation by lice or nits, through direct visual exam and the use of a metal microchanelled fine-toothed comb.

RESULTS: The visual diagnosis detected infestation in $30.7 \%$ of the cases, while the metal comb detected infestation in $51.5 \%$. Females were the most affected. The forms of parasites detected through direct visual exam were: only lice (adults and/or nymphs) 1.4\%, only live nits $64.8 \%$ and live nits and lice, $33.8 \%$; with the metal comb the percentages were $6.4 \%, 23.6 \%$ and $70 \%$, respectively. The average time to find a louse was 57 seconds with the fine-toothed comb and 116.4 seconds through the direct visual exam.

Conclusions: Diagnosis with the microchanelled fine-toothed comb is twice as fast and 3.6 times more efficient than through direct visual exam. The direct visual exam detects non-active, past infestations, and underestimates active ones.
\end{abstract}

Key words: Chile; lice infestations; Pediculus capitis; scalp dermatoses

Recebido em 11.08.2008

Aprovado pelo Conselho Consultivo e aceito para publicação em 08.12.08

* Trabalho realizado pela Cátedra de Parasitologia, del Departamento de Preclínicas, Escuela de Medicina, Facultad de Medicina, Universidad de Valparaiso, Valparaíso. Chile. Trabalho premiado como pôster de Investigação no Congresso de Dermatologia de 2008.

Conflito de interesse: Nenhum / Conflict of interest: None

Suporte financeiro: / Financial funding: Proyecto DIPUV 12-2002-2004; Escuela de Medicina, Facultad de Medicina, Universidad de Valparaiso, Valparaíso. Chile y Fundação Children International Chile, V Região.

Mestrado em Ciências Biológicas Menção Parasitologia. Professora Titular e Chefe da Cátedra de Parasitología, Departamento de Preclínicas. Escuela de Medicina, Facultad de Medicina, Universidad de Valparaíso, Valparaíso. Chile.

Médico clínico geral. Residente de Ginecologia, Hospital Carlos van Buren, Valparaíso - Chile.

Médica clínica geral, Hospital de Quillota - Chile.

Técnico de Laboratório. Cátedra de Parasitología, Departamento de Preclínicas. Escuela de Medicina, Facultad de Medicina, Universidad de Valparaiso, Valparaíso. Chile.

Médico clínico geral. Pós graduando do Instituto de Dermatologia Professor Rubem David Azulay da Santa Casa de Misericórdia do Rio de Janeiro. Professor associado da Cátedra de Parasitología, Departamento de Preclínicas. Escuela de Medicina, Facultad de Medicina, Universidad de Valparaiso, Valparaíso. Chile. 


\section{INTRODUÇÃO}

Entre os artrópodes ectoparasitas permanentes do homem encontra-se o piolho da cabeça Pediculus humanus var. capitis (Insecta: Phthiraptera), inseto estenoxênico (adaptado a uma espécie), ${ }^{1}$ de hábitos hematófagos, que desenvolve todo o seu ciclo de vida sobre o hospedeiro.

A pediculose é uma doença que tem afetado a humanidade ao longo de toda a história. ${ }^{2,3}$ Sua alta prevalência foi relacionada com a limitada disponibilidade de água e a deficiências nas práticas higiênicas, ${ }^{4}$ situação que diminuiu na primeira metade do século $\mathrm{XX}$, pela melhora em ambas as situações. Porém, nos anos 60 , houve um recrudescimento significativo na prevalência mundial da pediculose,$^{2}$ fato que foi relacionado às novas características dos modos de vida de certas subculturas nesse momento, tais como: o declínio dos hábitos de higiene pessoais, o aumento da promiscuidade sexual e o uso de cabelos longos, principalmente, entre a população jovem. ${ }^{4}$

No Chile, assim como em muitos outros países, essa parasitose não é uma doença de notificação obrigatória e não se mantém uma vigilância ativa que permita conhecer as taxas de infestação na população infantil. Na segunda metade da década de 90, trabalhos realizados em estudantes do Ensino Fundamental em Santiago do Chile, provenientes dos setores norte, leste e oeste da cidade, mostraram prevalências de 14,8\% nos homens e 31,6\% nas mulheres; os provenientes do setor sul da capital exibiam prevalências de $10,9 \%$ nos homens e de $79,2 \%$ nas mulheres. ${ }^{2} \mathrm{Na}$ região de Valparaíso (que se limita, ao norte, com a capital do País), os estudos de prevalência em escolares de procedência rural mostraram diferenças de $0,8 \%$ a $46,8 \%,{ }^{5}$ sendo maior em crianças oriundas de áreas suburbanas $(52,4 \%)^{6}$ e inferior em adultos hospitalizados em instituições psiquiátricas $(7,2 \%){ }^{7}$

A maior parte dos estudos em pediculose utiliza para o diagnóstico a técnica de exame visual direto para identificar os parasitas, ${ }^{8-12}$ a qual se caracteriza pela procura manual e observação a olho nu das diferentes fases do parasita. Em um paciente infestado existe maior número de lêndeas do que de pedículos (imagos e/ou ninfas), razão pela qual a pesquisa diagnóstica inicial se realiza, na prática, pela visualização das primeiras. ${ }^{10}$

Por outro lado, existem diferentes tipos de pentes finos: de plástico, alumínio, aço, termais ou eletrônicos, os quais são usados e recomendados como complemento do tratamento médico, ${ }^{13}$ porém, sua eficácia para o diagnóstico não tem sido avaliada. $\mathrm{O}$ pente metálico de aço, cujo fabricante indica como sendo apropriado para a extração de lêndeas, não tem sido utilizado com finalidades diagnósticas, mas a adequada separação entre seus dentes, a sua resistência e o fato de ser microcanaliculado, o que permitiria remover lêndeas e imagos com maior facilidade pelo arraste, fariam dele o método ideal de diagnóstico. Por todas essas razões e por não existirem estudos sistemáticos sobre qual seria o melhor método para certificar o diagnóstico, o objetivo do presente trabalho foi comparar a eficácia do exame visual direto versus o uso do pente metálico de aço com dentes microcanaliculados Assy 2000 para o diagnóstico de Pediculus bumanus var. capitis.

\section{MATERIAL E MÉTODOS Pentes}

O pente metálico com dentes microcanaliculados Assy 2000 (Laboratório Andrómaco) (Figura 1) é fabricado totalmente em aço inoxidável e pesa 42 gramas. É constituído por um cabo arredondado de $65 \mathrm{~mm}$ de diâmetro (com quatro aplicações de borracha antideslizante em ambos os lados), para o manuseio do pente, a cuja superfície estão unidos 33 dentes (de $38 \mathrm{~mm} \times 1,5 \mathrm{~mm}$ ) de aço circulares e canaliculados. Esses dentes terminam em pontas microscopicamente arredondadas, que objetivam proteger $o$ couro cabeludo de ferimentos.

\section{Pacientes}

A população total examinada correspondeu a 946 crianças e adolescentes, sendo 489 do sexo feminino e 457 do sexo masculino, entre quatro e 19 anos, que pertenciam aos registros de apadrinhamento da fundação Children International-Chile da cidade de Quillota (latitude $-32,88^{\circ}$ e longitude $-71,25^{\circ}$ ), Província de Valparaíso, Chile, e que foram citadas pelas assistentes do programa de saúde daquela insti-

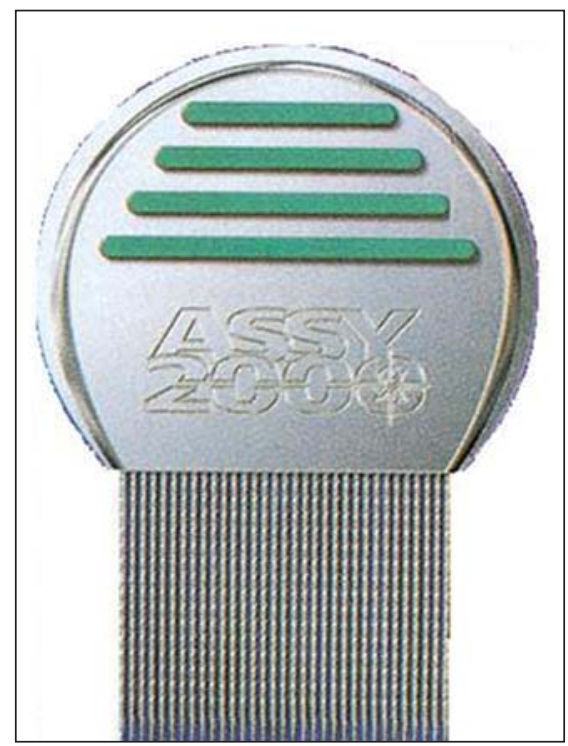

Figura 1: Pente metálico de aço inoxidável com dentes microcanaliculados Assy 2000 
tuição. A realização do estudo contou com o apoio de monitores de saúde da fundação, que foram previamente capacitados de forma teórico-prática pela Cátedra de Parasitologia da Escola de Medicina da Universidade de Valparaíso, ${ }^{14}$ com o objetivo de envolver e sensibilizar a comunidade local com o problema de saúde em pauta.

A equipe examinadora protegeu-se com as seguintes medidas de biossegurança: uso de avental branco com elástico nos punhos e um peitilho plástico que cobria desde o peito até os joelhos; os cabelos das participantes do sexo feminino foram amarrados; em ambos os sexos, os cabelos foram protegidos por touca ou turbante. Apesar de este trabalho ter sido desenvolvido no âmbito do programa de saúde da fundação, a cada pai e/ou responsável se solicitou consentimento para a participação das crianças e adolescentes, após receber informações sobre os objetivos do estudo e as atividades que seriam realizadas.

Examinou-se cada criança ou adolescente duas vezes, tanto pela observação visual direta quanto pelo uso do pente metálico microcanaliculado. Os cabelos de cada um foram desenrolados previamente com um pente de plástico de dentes grandes e separados, para evitar tração excessiva e, assim, facilitar o diagnóstico pelos dois métodos. Para o exame visual direto, utilizaram-se condições de luz adequadas, de modo a permitir o diagnóstico (Figura 2). A procura de parasitas iniciou-se na região occipital e retroauricular, estendendo-se, em seguida, para o resto da cabeça. Os cabelos foram separados com os dedos das mãos para se detectarem imagos, estados ninfais, lêndeas vivas ou mortas. Posteriormente, outro examinador (localizado em uma sala diferente) utilizou o pente metálico

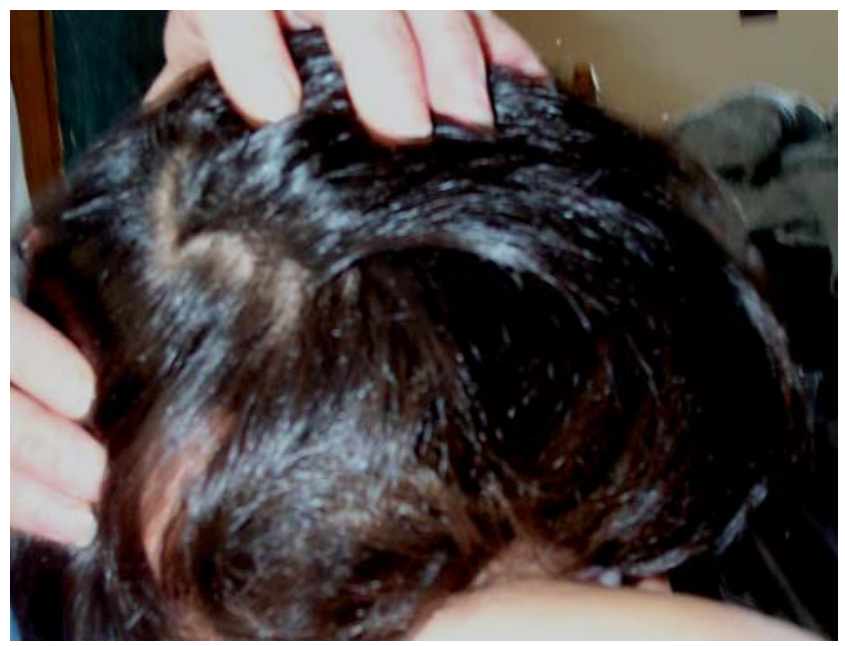

Figura 2: Exame visual direto para o diagnóstico de Pediculus bumanus var. capitis
(Figura 3), o qual foi passado desde a base dos cabelos até as pontas, para retirar por arraste qualquer forma parasitária que estivesse neles. O conteúdo do pente foi esvaziado em uma pequena bandeja branca, para se poderem diferenciar os parasitas de qualquer outro tipo de sujeira que o pente tivesse arrastado. Quando necessário, utilizou-se uma lupa com a finalidade de diferenciar as lêndeas vivas das mortas e de qualquer outro tipo de resíduo morfologicamente similar a olho nu. Uma vez finalizado o exame, fez-se a descontaminação com água fervente das bandejas de depósito, do pente desenrolante plástico e do metálico, passando-se neste último uma pequena escova com dentes finos, para eliminar qualquer tipo de forma parasitária e/ou resíduos. Posteriormente, secaram-se todos os instrumentos com ar quente. $\mathrm{O}$ tempo de detecção do primeiro piolho foi medido com um cronômetro por outro integrante da equipe e anotado junto a outras informações. Os dados obtidos foram registrados em uma planilha (Excel) e analisados pelo departamento de estatística da universidade por meio do programa Stata versão 8.0 (Statacorp, 2004) e realizando o teste de qui-quadrado, com um nível de significância de 5\% e determinação do risco relativo (odds ratio).

\section{RESULTADOS}

O registro de apadrinhamento da Fundação Children International-Chile da cidade de Quillota, Província de Valparaíso, corresponde a 1.061 crianças e adolescentes entre quatro e 19 anos de idade. Neste estudo, $89,2 \%$ de todos os pais ou responsáveis receberam as informações e/ou deram seu consentimento (o que resultou em um total de 946 menores examinados).

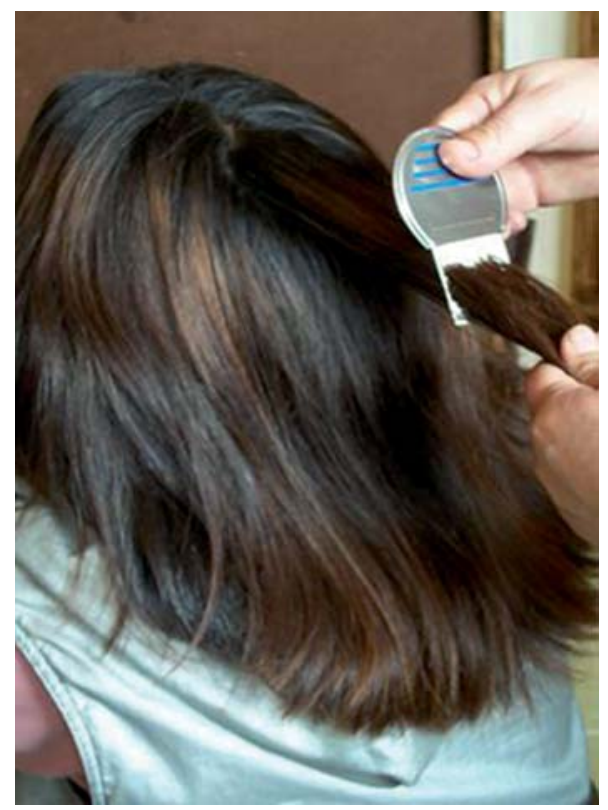

Figura 3 Exame com pente metálico microcanaliculado para diagnóstico de Pediculus capitis 
A observação da prevalência da infestação por Pediculus capitis mediante o uso do pente metálico com dentes microcanaliculados foi de $51,5 \%$ em média, variando entre $27,8 \%$ e $70 \%$ nos diferentes subsetores. Em comparação, a prevalência observada por meio do exame visual direto foi de $30,7 \%$, com uma variação zonal entre $7,4 \%$ e $47,7 \%$ (Tabela 1 ). Vale a pena destacar que todos os casos positivos ao exame visual foram corroborados pelo uso do pente metálico.

Ambas as técnicas apontaram maior incidência no sexo feminino. A positividade foi de $64,7 \%$ nas meninas e $35,3 \%$ nos meninos com o pente metálico e de $65,9 \%$ e $34,1 \%$, respectivamente, com o método visual direto.

Quanto aos estádios do Pediculus humanus var. capitits encontrados, pode-se dizer que, por meio do exame visual direto, foram detectados: somente piolhos (estados ninfais e/ou adultos) em 1,38\%; somente lêndeas vivas em $64,8 \%$ dos casos e piolhos + lêndeas vivas em 33,8\% dos casos (Gráfico 1). As formas achadas pelo pente metálico foram: somente piolhos em 6,4\% dos casos; somente lêndeas vivas em 23,6\% da amostra e, finalmente, piolhos + lêndeas vivas em $70 \%$ dos casos (Gráfico 2). Lêndeas mortas foram encontradas em 389 casos, com o exame visual direto, e em 541 casos com o uso do pente metálico, resultados que não foram considerados diagnósticos. O gráfico 3 apresenta uma comparação entre ambos os métodos quanto às formas parasitárias detectadas.

A análise estatística indicou que o pente metálico detectou o piolho adulto, em média, 213 mais vezes do que o método visual direto, com odds ratio $=161$ e IC $=70-368$. No sexo masculino, o pente metálico permite encontrar 146 mais vezes o piolho adulto do que o método visual direto. No sexo feminino, esse valor passa para 249 vezes, 3,64 vezes maior que nos homens.

A média de tempo para a detecção do primeiro piolho na cabeça de um paciente examinado foi de 57 segundos com o pente metálico e de 116,4 segundos pelo exame visual direto.

\section{DISCUSSÃO}

No Chile, tem ocorrido uma tendência a subestimar a pediculose por Pediculus bumanus var. capitis, já que, classicamente, esse parasita é visto como não sendo vetor de microorganismos patogênicos que causem outras doenças. ${ }^{2}$ Atualmente, essa é uma questão controvertida, uma vez que Murray e Torrey conseguiram infectar experimentalmente Pediculus humanus var. capitis com Rickettsia prowazekii, agente etiológico do tifo epidêmico, ${ }^{15}$ e Sasaki et al. isolaram Bartonella quintana desde pedículos, que causa a febre das trincheiras (trench fever). ${ }^{16} \mathrm{O}$
Pediculus capitis pode parasitar indivíduos de diversas condições econômicas, porém, em nosso meio, há uma tendência generalizada a se acreditar que esse parasita se distribuiria, principalmente, entre pessoas de classes mais desprotegidas e com práticas higiênicas deficitárias. A prevalência global de 51,5\% detectada na cidade de Quillota, na Província de Valparaíso, Chile, confirma que essa parasitose se mantém em elevada percentagem da população, evidenciando que a doença continua sendo um problema de saúde pública e um indicador de nível cultural deficiente.

O método diagnóstico classicamente utilizado pelos diferentes estudos epidemiológicos e clínicos tem sido a visualização direta dos piolhos (adultos e estados ninfais) ou, principalmente, das lêndeas localizadas perto do couro cabeludo. Por outro lado, a recomendação para o uso dos diversos tipos de pentes de dentes finos é, fundamentalmente, uma medida coadjuvante no tratamento medicamentoso, ${ }^{17}$ tendo sido descrita sua utilização com finalidades diagnósticas em apenas um trabalho. De Maeseneer et al. $(2000)^{18}$ compararam em 224 pacientes o uso de um pente plástico de dentes finos em cabelo molhado em contraposição à visualização direta a olho nu. A experiência apresentou melhores resultados com o pente, mas são necessários mais estudos para validar a técnica. Desde então, nada foi publicado na literatura mundial sobre essa questão, o que evidencia, uma vez mais, o pouco interesse existente sobre o tema da pediculose.

A grande diferença na prevalência encontrada a partir da avaliação com pente metálico de dentes microcanaliculados $(51,5 \%)$ versus o método visual direto $(30,7 \%)$ confirma a grande utilidade do pente em matéria de diagnóstico (Tabela 1). O melhor rendimento diagnóstico obtido pelo pente metálico pode se dever à estrutura deste: a separação entre os dentes do pente $(0,094 \mathrm{~mm})$, ao ser menor que o tamanho das lêndeas $(0,2 \mathrm{~mm})$ e dos piolhos $(0,7 \mathrm{~mm})$, permitiria seu arraste desde a base do cabelo até a ponta e, assim, sua eliminação. O comprimento dos dentes $(38 \mathrm{~mm})$ aumentaria a área útil do arraste, facilitando a retirada de mais lêndeas em menos passadas e garantindo sua eficácia em todo tipo de cabelo: comprido, curto, liso, crespo, fino, grosso ou abundante. Ao ser resistente à flexão, o pente desliza sem provocar muita tensão sobre a haste, o que é importante nos cabelos crespos. Além disso, o pente apresenta outras vantagens: pontas arredondadas, que evitam o trauma sobre o couro cabeludo; bandas de borracha antideslizantes, que permitem uma boa manipulação do instrumento; finalmente, sua composição à base de aço inoxidável permite uma constante desinfecção com água fervente, fato importante a considerar no momento de fazer avaliações comunitárias. Por outro 
TABela 1: Comparação da infestação por Pediculus humanus var. capitis pelo método visual direto e pelo pente metálico com dentes microcanaliculados em crianças e adolescentes dos subsetores da cidade de Quillota, Província de Valparaíso, Chile

\begin{tabular}{|c|c|c|c|c|c|}
\hline \multirow{3}{*}{ Subsetor } & \multicolumn{4}{|c|}{ Método } & \\
\hline & \multirow{2}{*}{$\begin{array}{l}\text { Indivíduos } \\
\text { avaliados }\end{array}$} & \multicolumn{2}{|c|}{ Visual direto } & \multicolumn{2}{|c|}{ Pente metálico } \\
\hline & & $\mathbf{N}^{\circ}$ de casos & $\%$ & $\mathbf{N}^{\circ}$ de casos & $\%$ \\
\hline Aconcagua & 156 & 59 & 37,8 & 81 & 51,9 \\
\hline Bajío & 20 & 7 & 35 & 14 & 70 \\
\hline Boco & 77 & 20 & 26 & 33 & 42,9 \\
\hline Cajón San Pedro & 18 & 3 & 16,7 & 5 & 27,8 \\
\hline Cerro Mayaca & 118 & 36 & 30,5 & 59 & 50 \\
\hline El Esfuerzo & 109 & 38 & 34,9 & 62 & 56,9 \\
\hline El Mirador & 30 & 9 & 30 & 18 & 60 \\
\hline Enrique Arenas & 18 & 3 & 16,7 & 8 & 44,4 \\
\hline Las Pataguas & 22 & 9 & 40,9 & 12 & 54,5 \\
\hline Lo Varela & 21 & 5 & 23,8 & 6 & 28,6 \\
\hline Logarzo & 90 & 24 & 26,7 & 47 & 52,2 \\
\hline Los Almendros & 38 & 8 & 21,1 & 22 & 57,9 \\
\hline Los Lucumos & 44 & 21 & 47,7 & 30 & 68,2 \\
\hline Manuela Figueroa & 70 & 22 & 31,4 & 41 & 58,6 \\
\hline Pocochay & 27 & 2 & 7,4 & 9 & 33,3 \\
\hline Pueblo Indio & 23 & 5 & 21,7 & 10 & 43,5 \\
\hline San Pedro Centro & 21 & 6 & 28,6 & 11 & 52,4 \\
\hline Sol de Julio & 16 & 4 & 25 & 7 & 43,8 \\
\hline Villa El Esfuerzo & 28 & 9 & 32,1 & 12 & 42,9 \\
\hline Total & 946 & 290 & 30,7 & 487 & 51,5 \\
\hline
\end{tabular}

lado, a diferença relevante encontrada entre os dois métodos levanta um sério questionamento quanto à real prevalência da pediculose em diversos lugares do mundo, baseada em estudos que têm utilizado apenas o exame visual direto.

O diagnóstico seguro fundamenta-se no achado de formas adultas ou juvenis do parasita, o que não ocorre com muita frequência mediante o exame visual direto, à exceção de infestações maciças, devido ao fato de as formas adultas e ninfais serem fotofóbicas e, portanto, terem a tendência a se refugiar na espessura dos cabelos ao mexer-se neste (no intento de fazer o diagnóstico por meio do exame visual direto). Existe, então, maior probabilidade de detectar a pediculose em lêndeas, visto que se apresentam como pequenas formações ovóides firmemente aderidas à base dos cabelos. As situações assinaladas anteriormente são corroboradas por nossos resultados (Gráficos 1, 2, 3). As lêndeas situadas a mais de $6 \mathrm{~mm}$ do couro cabeludo não são viáveis, pois eclodem de cinco a 10 dias após sua postura. Como os cabelos crescem entre 10 e $32 \mathrm{~mm}$ por mês, esse fato serviria de base para diagnosticar infestações não ativas quando se encontrassem apenas lêndeas em um indivíduo.
A relação entre comprimento do cabelo e risco de infestação é um tema controvertido. Muitos textos negam essa relação, atribuindo pouco valor a esse aspecto como elemento favorecedor ou perpetuador da infestação, ${ }^{13}$ porém, outros trabalhos apontam uma

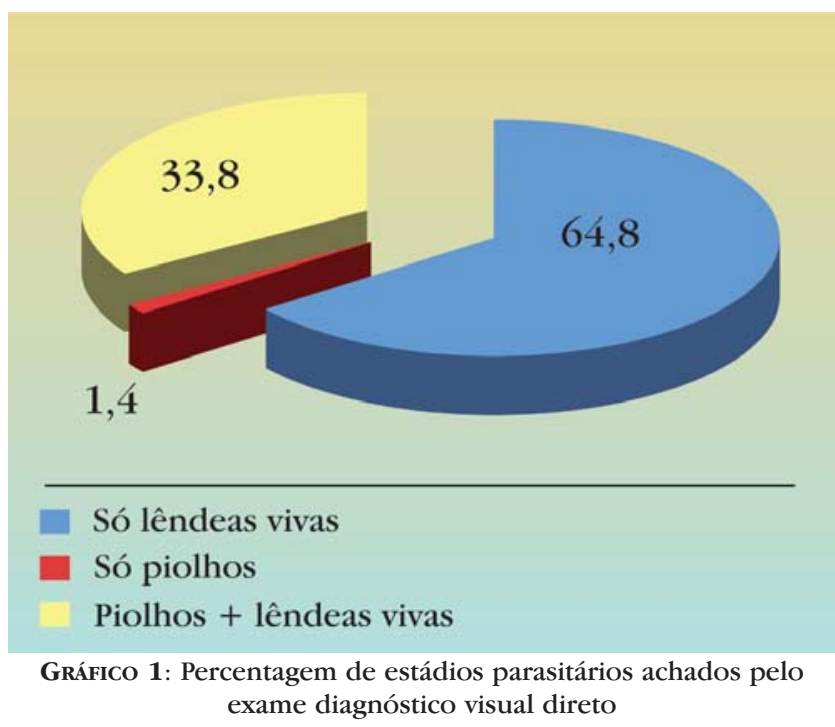




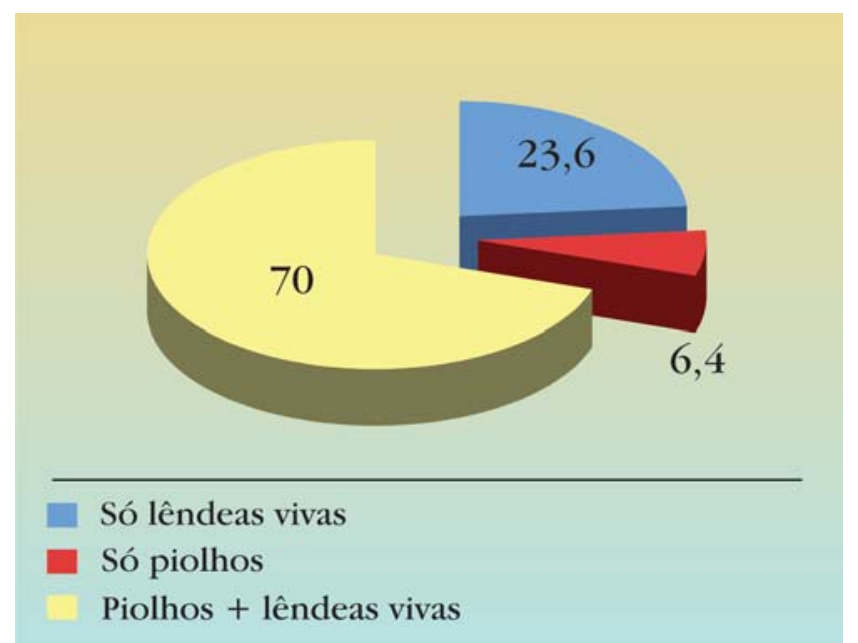

Gráfico 2: Percentagem de estádios parasitários achados mediante pente metálico

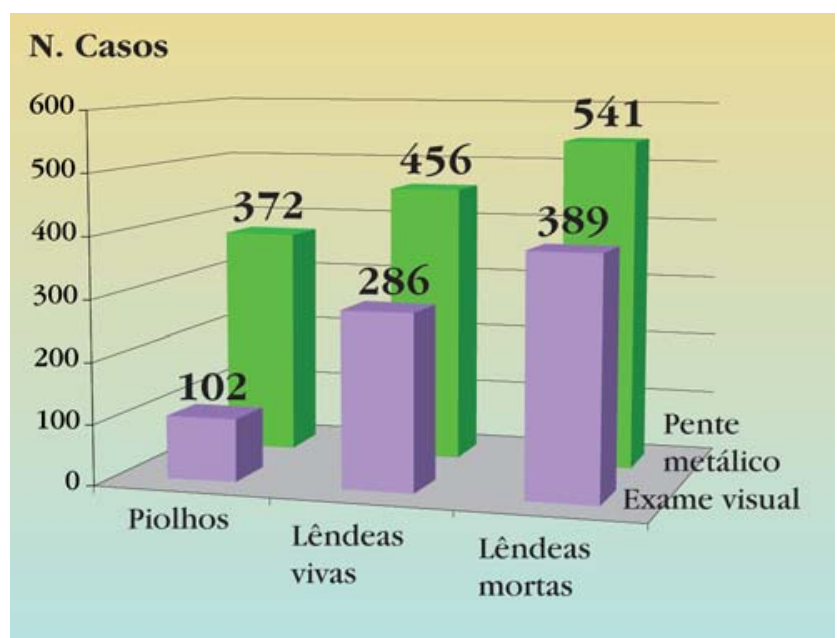

Gráfico 3: Comparação das formas parasitárias detectadas pelo método visual direto e o pente metálico com dentes microcanaliculados

A grande prevalência de Pediculus capitis, seu impacto na saúde pública e o evidente predomínio na população estudada justificam amplamente que o tema seja enfocado como um problema médico-sanitário e sua profilaxia seja planejada seriamente. É fundamental fazer a comunidade participar de seus problemas de saúde, em especial, os adultos responsáveis pelos menores, como foi no caso do estudo, os quais, por ignorância ou negligência, mantêm essa situação. Em muitos casos, observamos o enfrentamento da pediculose, por parte do grupo estudado, com muitos mitos e práticas infundadas e de alto risco (dados não publicados).

\section{CONCLUSÃO}

O método visual direto subestima as infestações ativas e detecta as já passadas não ativas. A partir da alta prevalência encontrada (51,5\%), é possível afirmar que o diagnóstico da infestação por Pediculus capitis é duas vezes mais rápido e 3,6 vezes mais eficiente com o pente metálico de dentes microcanaliculados, razões que o confirmam como um método sensível e reprodutível, de grande utilidade para estudos epidemiológicos e na prática diária do médico. 


\section{REFERÊNCIAS}

1. Downs AM, Stafford KA, Cole GC. Head lice: prevalence in schoolchildren and insecticide resistance. Parasitol Today. 1999;15:1-4.

2. Schenone H, Lobos M. Pediculosis capitis, un permanente y renovado problema. Bol Chile Parasitol. 1997;52:73-6.

3. Mumcuoglu KY. Prevention and treatment of head lice in children. Paediatr Drugs. 1999;1:211-8.

4. Schenone H, Saavedra T, Rojas A. Infestación por Pediculus bumanus capitis. Un prolongado problema de salud pública. Bol Chile Parasitol. 1986;41:16-20.

5. Neira P, Goecke I, González W, Muñoz N.

Ectoparasitosis en escolares rurales de la $5^{\text {a }}$ Región, Valparaíso, Chile - 1986. Bol Chile Parasitol. 1987;42:87-9.

6. Neira P, Arenas C, Neira G, Valenzuela N, Subercaseaux B. Infecciones parasitarias en niños de una escuela de Reñaca Alto. Rev Med Valparaíso. 1981;34:56-60.

7. Garibaldi R, Muñoz N, Neira P, Subercaseaux B, Villalón L. Entero y ectoparasitosis en la $5^{\mathrm{a}}$ Región, Chile. Estudio en el Hospital Psiquiátrico de Putaendo. Bol Chile Parasitol. 1990;45:83-5.

8. Cazorla D, Ruiz A, Acosta M. Estudio clínico-epidemiológico sobre pediculosis capitis en escolares de Coro, estado Falcón, Venezuela. Invest Clin. 2007;48:445-57.

9. Milano AF, Oscherov EB, Legal AZ. Pediculosis y otras ectoparasitosis en una población infantil urbana del nordeste argentino. Parasitol Latinoam. 2007;62:83-8.

10. Borges R, Silva JJ, Rodrigues RM, Mendes J. Prevalence and monthly distribution of head lice using two diagnostic procedures in several age groups in Uberlândia, State of Minas Gerais, Southeastern Brazil. Rev Soc Bras Med Trop. 2007;40:247-9.

11. Catalá S, Junco L, Vaporaky R. Pediculus capitis infestation according to sex and social factors in Argentina. Rev Saúde Pública. 2005;39:438-43.

12. Calderón-Arguedas $\mathrm{O}$, Solano $\mathrm{M}$, Sánchez $\mathrm{C}$. El problema de la pediculosis capitis en escolares del área metropo litana de San José, Costa Rica. Parasitol Latinoam. 2003;58:177-80.
13. Speare R, Canyon DV, Cahill C, Thomas G. Comparative efficacy of two nit combs in removing head lice (Pediculus humanus var. capitis) and their eggs. Int $\mathrm{J}$ Dermatol. 2007;46:1275-8.

14. Neira P, Undurraga O, Muñoz N, Silva P, Saínz M, Molina L. Capacitación sobre pediculosis en líderes comunitarias. Anuario Escuela de Medicina Universidad de Valparaíso. 2003;7:217-24.

15. Murray ES, Torrey SB. Virulence of Rickettsia prowazekii for head lice. Ann N Y Acad Sci. 1975;266:25-34.

16. Sasaki T, Poudel SK, Isawa H, Hayashi T, Seki N, Tomita T, et al. First molecular evidence of Bartonella quintana in Pediculus humanus capitis (Phthiraptera: Pediculidae), collected from Nepalese children. J Med Entomol. 2006;43:110-2.

17. Rosso RO, Ramírez MS, Torres M. Pediculus capitis: terapias disponibles. Rev Chil Infect. 2003;20:111-6.

18. De Maeseneer J, Blokland I, Willems S, Vander Stichele $\mathrm{R}$, Meersschaut $\mathrm{F}$. Wet combing versus traditional scalp inspection to detect head lice in schoolchildren: observational study. BMJ. 2000;321:1187-8.

19. Schenone H, Falaha F, Villarroel F, Rojas A, Szekely R, Rojo M, et al. La infestación por Pediculus humanus capitis en Santiago de Chile. Bol Chil Parasitol. $1973 ; 28: 31-3$.

ENDEREÇO PARA CORRESPONDENCIA / MAILING ADDRESS:
Patricia E. Neira.
Cátedra de Parasitología. Departamento de
Preclínicas. Escuela de Medicina, Facultad de
Medicina, Universidad de Valparaíso, Hontaneda
2653-Valparaíso, Chile PO Box 92-V.
Valparaíso Chile
Fono: 56-32-507349, Fax: 56-32-507321.
E-mail: patricia.neira@uv.cl

ENDEREÇO PARA CORRESPONDÊNCIA / MAILING ADDRESS:

Patricia E. Neira. Medicina, Universidad de Valparaíso, Hontaneda 2653-Valparaíso, Chile PO Box 92-V.

E-mail: patricia.neira@uv.cl

Como citar este artigo / How to cite this article: Neira PE, Molina LR, Correa AX, Muñoz NRA, Oschilewski DE. Utilidade do pente metálico com dentes microcanaliculados no diagnóstico da pediculose. An Bras Dermatol. 2009;84(6):615-21. 